\title{
Exploring some aspects that drive the global perception of the facial appearance of young Caucasian adult men by a panel of Caucasian women of same ages
}

\begin{abstract}
Objective: To decipher, through different wordings, the perception of adult men faces by "naïve" panels of women, through two different photographic modes (standard \& natural) and its possible link with some objective/ instrumental assessments.

Methods: The full faces of 118 men (aged 30-45y) were photographed under a standardized mode (hiding hairs and upper garments) and under a natural look, showing hairstyles and upper garments. 40 of these men were bearded and were photographed (standardized mode) pre and post shaving. 260 "naïve" women of same age-range, divided in three groups, were asked to express their feelings about the psycho-social attributes of these 118 men and their hedonistic appreciation through spontaneous verbatims. A quiz part, using fixed wordings, graded through a $0-10$ scale, allowed to confer "quantitative" assessments on the various psycho social and the perceived behavior of these men. These gradings were analyzed through Principal Component Analysis (PCA).The facial skin of the 118 men was clinically assessed and instrumentally investigated, focusing on wrinkles/folds, sagging/ptosis, color, hydration and plumpness/water mobility.
\end{abstract}

Results: A Hierarchical Cluster Analysis allowed to distinct two different clustering on the psycho-social perceptions according to the photographic mode ( 8 in standard and 6 in natural conditions), based on the different frequencies of the quoted verbatims.

Shaving the beard led to many individual shifts (of various amplitudes) within both psycho-social and behavioral appreciations. When shaved, these 40 men were globally seen as more "neat", more intellectual and younger by about one year. However, shaving does not lead to clear shift within the psycho-social clusters.

\section{Introduction}

Facial appearance is a paramount driving factor in individual and social relationships, ${ }^{1-5}$ including the perception of age, i.e. older, younger. ${ }^{6-9}$ This human need is probably as old as humanity, witnessed by prehistoric sculptures or Egyptian make-ups, extravagant adornments in the 18th century in Europe. In full logic, the possible external and provisory modification(s) of facial appearance, have been largely studied by cosmetic researchers, mostly focusing on women(approx. $90 \%$ of their consumers). ${ }^{10-12}$ Unsurprisingly, a large majority of cosmetic products are dedicated to the feminine face and its head hair (skin care, sun-protection, foundations, mascaras, lipsticks, hair styling, dying, perming.).

The inverse scenario, i.e. the appearance of men's faces, appears scarcely studied, in line with the rather limited market of cosmetic products dedicated to men, in developed countries. This aspect was initially approached by 4 referential Atlases where 15 to 20 signs of facial ageing of both genders, from four ethnics (AfricanAmerican, Asian, Caucasian, Indian) were described through standardized photographs. ${ }^{13-16}$ The latter allowed to attribute a
Volume 3 Issue 4 - 2019

\author{
Frederic Flament,' David Amar, ${ }^{2}$ Aurelie \\ Decroux,' Celine Cornillon, ${ }^{3}$ Anne-Sophie \\ Adam, ${ }^{3}$ Jonas Morel, ${ }^{4}$ Aude Charbonneau ${ }^{4}$ \\ 'L'Oréal Research and Innovation, Clichy, France \\ 'L'Oréal Research and Innovation, Shanghai, China \\ ${ }^{3}$ L'Oréal Research and Innovation, Chevilly-Larue, France \\ ${ }^{4}$ Eurosyn, Villebon-sur-Yvette, France
}

Correspondence: Flament Frederic, L'Oréal Research and Innovation, Clichy, France, Tel +33I49795334,

Email frederic.flament@rd.loreal.com

Received: August 01, 2019 | Published: August 19, 2019

Men of some clusters defined on natural pictures have been found significantly different from others based on several objective parameters.

Conclusion: The facial appearance of Caucasian men by Caucasian women is strongly driven by cultural, behavioral or social perceptions and less by men's facial signs and skin properties. These appear much influenced by the visions of hair or garments, as well as presence of a beard.

Keywords: Facial signs, Caucasian men, Tribes, Facial appearance, Perception

grading score according to the severity of each facial sign (structures or pigmentations), showing that changes in facial signs with age $(18$ to $>70 y)$ do not present comparable kinetics and differ between ethnics. ${ }^{17}$ Although highly useful in clinical grinding's, these Atlases remain however "neutral" references, that depicts facial signs taken separately. The global facial picture is thus ignored, although being paramount in the spontaneous facial recognition by "the others" the brain of which integrates, at a glance, all facial signs. These Atlases are by nature deprived from any personal appreciations, i.e. free from subjective or cultural criteria that are commonly and daily used by all of us.

The male facial appearance remains nonetheless a less studied topic, although cleverly approached by studies of Fink and coll., based on objective measurements and their related interpretations. ${ }^{18-20}$ The human facial appearance (the complex concept of "beauty"), as recently reviewed, ${ }^{21}$ is indeed a multi-parametric criterion (subjective and objective) that largely encompasses skin and hair criteria.

Aside from factual/objective data, little is known how, and through which wordings, the faces of men can be spontaneously and 
subjectively perceived by women through their imagined individual, behavioral criteria or social status, leading to a variable attractiveness.

Accordingly, as a first and exploratory approach, a study was designed and conceived on a restricted panel of 118 Caucasian men, aged $30-45 y$, where face was first photographed, then objectively measured and therefore assessed by 260 "naïve" Caucasian women (i.e. unaware of cosmetic related issues) of a similar age-range than that of the118 men to avoid possible different age/cultural/fashionrelated bias.

Based on three types of digital photographs of the faces, these women were asked to assess, through fixed verbatim, their subjective and spontaneous perception of these 118 men. The three types of blind-coded photographs comprised: i) full face where hairs and top garments were artificially hidden (referred as standard), ii) full face where head hairs and top garments were visible (referred as natural). Among these 118 men, 40 wore a beard, and two series of photographs were taken (standard mode) pre and post shaving. Based on the spontaneous clusters definition on natural pictures and objective clinical or instrumental measurements, the decoding of the perception of "tribes" could be investigated.

The results of this purely subjective and exploratory study are the objects of the present paper.

\section{Material and methods}

\section{Subjects}

118 young adult healthy Caucasian men (aged 30-45y), living in the Parisian area, were recruited from agencies dedicated to part-time occupations. All subjects represented all socio professional categories and were full time employees. For sake of confidentiality, their marital and parental status was not considered. When present, a too developed beard (a few cms) was carefully excluded, as these men were unlikely willing to shave. A light beard, i.e. of a few days, was given privilege.

Inclusion criteria were the following:

No sun exposure (or UVA cabin) 2 weeks prior to the study.

No application of sun-free tanning product 2 weeks and skin care products 2 days, prior to the study.

Not shampooing the evening before and the starting day of the study.

Absence of practice of swimming pool, sauna or hammam the very day of the study.

Being shaved at least 2 hours prior to photographic shootings (for non-beard subjects only).

Bearded subjects accepting to being mechanically/electrically shaved the very day of the photographic shooting.

No previous facial aesthetic surgery.

No intake of warm beverages or spicy food, not smoking $1 \mathrm{~h}$ prior to the study.

All subjects were fully informed about the objective and the steps of the study (Figure 1) and signed an informed consent. As the right to imaging was not granted, they were ensured, by contract, that some possibly published photographs will be artificially eye-masked.



Figure I Global protocol of the study. 


\section{Pictures acquisitions}

All subjects enrolled in the study visited on a single occasion our local facilities in Paris. At arrival, the faces of the subjects were gently cleaned by cotton pads soaked with an Ethanol/Water solution $(70 / 30, \mathrm{v} / \mathrm{v})$ to eliminate traces of sebum or dirt, avoiding a shiny aspect. All men rested for 20 minutes under ambient conditions $\left(\mathrm{T}^{\circ}=21 \pm 1^{\circ} \mathrm{C}, \mathrm{RH} \%=45 \pm 5 \%\right)$ prior photographic shootings of their faces.

The faces of all 118 subjects, when dried-off, were photographed under standard conditions of lighting provided by a Bench Head Scan (Orion Concept), using a digital Nikon D300 digital camera fitted with a $105 \mathrm{~mm}$ objective (200 ISO sensitivity, 1/60 second, minimum aperture f/32) in a black room. Photographs were initially taken by masking all hair and top garments by a black cap and a black blouse and under natural conditions, where hair styles and part of the top garments become visible (shirt, sweater etc.). Subjects without beard were asked to shave the very morning of the photographic shootings and to adopt the most neutral expression during the photographic shooting. Due to the necessity of unmasking the hairs and top garments, standard and natural photographs presented different field sizes but similar to all paired-photographs. Photos 1 and 2 illustrate, as examples, the two photographic modes obtained on the same subject. The same procedures were applied to 40 bearded subjects (from the 118), i.e. photographed pre and post self-shaving, using a mechanic or electric razor, by standard mode only. Photos 3 and 4 illustrate, as examples, the same bearded subject with and without his beard.

\section{Evaluation and grading of facial skin aging signs on standard pictures}

All standard photographs of the 118 men were blind coded and reframed, aiming at focusing on different facial sites. The evaluation step consists in visualizing photographs by the panel of 15 trained experts for grading the severity of 7 facial signs according to the referential Skin Atlas specific to Caucasian men. ${ }^{13}$ Table 1 describes the facial signs under study for Caucasian men and their respective grading scales. These reference charts, according to the studied sign may vary between 0 (absent) and 5, 6 or 7 (severe) depending on the facial signs.

Table I 7 Facial signs related to Caucasian men and their photographic illustrations.

\begin{tabular}{|c|c|c|c|}
\hline Facial Signs & Definition of scored observation & Scale & Visual \\
\hline Forehead wrinkles & Depth of the transverse wrinkles on the forehead. & $0-6$ & \\
\hline Glabellar wrinkles & Depth of vertical wrinkles between eyebrows. & $0-7$ & \\
\hline Crow's feet wrinkles & $\begin{array}{l}\text { Depth of wrinkles at the area of outer eye corner }(5 \mathrm{~mm} \text { at least } \\
\text { outside from the corner). }\end{array}$ & $0-6$ & \\
\hline $\begin{array}{l}\text { Periorbital wrinkles (upper } \\
\text { cheek area) }\end{array}$ & Depth of folds at malar zone below Crow's feet, eye orbit excepted. & $0-6$ & \\
\hline Naso labial fold & $\begin{array}{l}\text { Depth of the folds present on face between the base of the nose } \\
\text { and lips. }\end{array}$ & $0-6$ & \\
\hline Marionette lines & Depth of folds at the corner of lips. & $0-5$ & \\
\hline $\begin{array}{l}\text { Ptosis of the lower part of the } \\
\text { face }\end{array}$ & Sagging severity of the lower parts of the chin. & $0-7$ & \\
\hline
\end{tabular}


The panel of 15 experts assessed all these signs through all photographs that were resized and reframed under Standard Operational Procedure with editing software (Photoshop version $10^{\circledR}$ ). This step ensured that a given sign was only displayed on the screen.

All pictures were presented to each expert at random, to eliminate bias. To ensure the robustness of this process, several pictures were presented twice during the evaluation process. The latter was performed under standardized conditions of lighting, position (expert being seated 1 meter from the screen) and calibration (24-inches, $1920 * 1200$-pixel high-resolution screen calibrated with a colorimeter). The final score of a given facial sign of each subject was therefore an averaged value calculated from the 15 experts' grading. ${ }^{17}$

\section{In vivo instrumental evaluation of facial skin}

To complete the clinical mapping of men's faces described above, some biophysical tools have been used by trained operator, after pictures shooting, on the 118 men enrolled in the study to approach different visible dimensions such as color, dryness or plumpness which could affect the perception of "others":

On jaw line, the DermoTrace ${ }^{\circledR}$ device $^{22}$ was used to assess the plumpness of tegument by applying a grid with a standardized pressure and analyze the pattern "imprinted" on skin after 30 seconds of application. This measure of water mobility could be linked with perception of "thick" or "thin" skin.

On lower cheek area three successive measurements were performed on three adjacent zones, using the Corneometer ${ }^{\circledR}$ CM 825 probe (Courage \& Khazaka, Koln, Germany) ${ }^{23}$ that quantifies the skin hydration.

A full-face image acquisition was performed with a Chromasphere ${ }^{\circledR}$ device (Monaderm, Monaco) ${ }^{11}$ and skin color parameters were measured on the cheek, using $\mathrm{L}^{*}, \mathrm{a}^{*}$ and $\mathrm{b}^{*}$ or the coxello index for homogeneity characterization. ${ }^{24,25}$

\section{"Naive" panel}

This panel comprised a total 260 women $(30-45 y)$ not affiliated or aware of the cosmetic/aesthetical domain. They were organized in three groups (two groups of 100 women each and one group of 60 women) that represented various socio-professional categories, with abnormal visual acuity, corrected by glasses or lenses when necessary. They were recruited under the same procedure used for the 118 subjects and were totally informed about the purposes of their task. The first group of the panel $(\mathrm{N}=100)$ was asked to observe 60 photographs in standard mode whereas the second group $(\mathrm{N}=100)$ observed the same subjects in natural mode to avoid possible intra recognition (i.e. same subject in both modes). Only 60 men were selected, among the 118, for: i) limiting the visual fatigue of the "naïve" panel, ii) respecting the age distribution of the whole cohort and iii) as the closest representatives of the shapes and volumes of the faces of the 118 men. These 200 women were then asked to express spontaneous wordings that best describe the men's personality, social rank, behavior, familial status (see below some verbatims used).

The third group of women ( $\mathrm{N}=60)$, was asked to evaluate the 158 photographs (118+40 with beard) on 15 pre-defined criteria. For this quiz part, all blind coded photographs were presented to the panel at random, in a closed black room, via a screen, the brightness and color of which were daily calibrated. Each criterion used (see below) was graded by the "naïve" panel through an analogic progressive $0-5$ scale according to a feeling of intensity ( 0 : Not at all, 1: Not, 2: A few, 3: A little, 4: almost, 5: Absolutely).

\section{Verbatim used for the sorting task}

Many different verbal French spontaneous expressions dealing with lifestyle, social position, general attitude etc. were recorded. Since numerous, these were further grouped into only one generic term that was thought best reflecting all synonyms or a consensual agreement. Table 2 summarizes the more frequent quoted terms expressed by the "naïve" panel (in French and their translated English equivalents) and the adopted generic. The latter will be used in the statistical analysis as possible referential axis.

\section{Quiz task}

Coupled to the visions of all 118 standardized photographs, a Quiz methodology used 10 fixed verbatims to which the third panel (60 women) had to answer through the $0-5$ scale previously mentioned. These were as follows:

Three other questions, based on a larger grading scale $(0-10,0$ : not at all, 10: very much) were the following:

Do you like/appreciate this man? (Hedonistic approach)

Does this man look tired?

Do you think this man has a sensitive skin?

In final, women were asked to give an apparent age to each man and to classify all these men into five psycho-social categories (Urban, Sensitive, Night Owl, Adventurous, Generous). Only one answer/ choice was accepted.

\section{Statistics}

For the sorting task:_2 types of information's were collected:

i)The appurtenance of each man to the groups previously created by the women. Both the number of groups chosen and the number of men within groups were let free to each woman.

A contingency table was thus created: the latter included the number of times $\mathrm{Mr} \mathrm{X}$ and $\mathrm{Y}$ were believed to belong to the same group ( $\min 0, \max 100)$. This table was further analyzed by MultiDimensional Scaling (MDS) to obtain a 2D cartography.

A Hierarchical Cluster Analysis (HCA), based on the coordinates of each man, was performed, allowing to gather the men that were more closely located on the map, within a same group.

ii)The "naïve" panel of women has additionally described groups with their own verbatims.

Assuming that a given word used to describe a group applies to all men comprised within this group, a table was created by crossing men*verbatim, i.e. through the different frequencies of quotes of each verbatim used to the same man. This table was further analyzed by Factorial Correspondence Analysis (FCA). The latter allowed to better interpret the previously established cartography.

For the Quiz part: Anova test was used to differentiate semiquantitative values (grades), taking a $\mathrm{p}<0.05$ as significant threshold. A Principal Component Analysis (PCA) was used for integrating men clusters with verbatims. As for the Sorting task, Hierarchical Cluster Analysis (HCA), based on the coordinates of each man, was performed, allowing to gather men that were more closely located on the map.

Clusters from natural pictures decoding based on objective parameters: The critical probability has been calculated through a 
t-test (bilateral-unpaired), comparing for each parameter the average of the cluster to the considered average of all the other clusters. Significance has been considered with a $5 \%$ level of risk. For sake of clarity and regarding size of studied population, only $p \leq 0.001$ statistical significance have been taking into account for facial sign and $\mathrm{p} \leq 0.01$ for instrumental assessments. Each significant parameters comes with its critical probability and the difference (in \%) between the average of the cluster and the one of all the other clusters.

All Statistics procedures were carried out by using XLStat ${ }^{\mathbb{}}$ (Addin soft SA, Bordeaux, France) or $\operatorname{Spad}^{\circledR}$ (Coheris SA, Suresnes, France).

Table 2 Examples of the verbatims used by the "naïve" panel (French expressions and their closest English translations), grouped under a common generic.

\begin{tabular}{|c|c|c|}
\hline FR Generic & ENG Generic & Comprised wordings \\
\hline Branché & Trendy & Fashion follower, Snob \\
\hline Extraverti & Extroverted & Cordial, Sociable, Empathic \\
\hline Introverti & Introverted & Retreat into oneself, Selfish, Egocentric \\
\hline Cadre installé & Executive Position & High job status, White Collar \\
\hline Sportif & Sportsman & Physically active, Team leader, Coach \\
\hline Mr Tout le monde & John Doe & Mr Such, Anonyms, passing by, Transparent \\
\hline Ecorché & Tormented & Bothered, Plagued, Taunted \\
\hline Dynamique & Dynamic & Active, Energetic, Enterprising \\
\hline Travailleur & Industrious & Hard working, Workaholic \\
\hline Patron & Boss & Wharfman, Conductor, Leader \\
\hline Tanguy & NEET (Not in Education, Employment or Training) & $\begin{array}{l}\text { Family cocoon oriented, Bachelor, still smothered by mom and } \\
\text { dad }\end{array}$ \\
\hline Père de Famille & Family Father & Pater Familias, Protector, Guardian \\
\hline Traditionnel & Classic & Conformist,Traditional, \\
\hline Doux & Gentle & Meek, Sweet, Tender \\
\hline Ambitieux & Ambitious & Careerist, Social climber, Arriviste \\
\hline Viril & Virile & Manly, Masculine, Red-blooded \\
\hline Sage & Wise & Discreet, Demure, Sober \\
\hline Fétard & Rounder & Roisterer, Merrymaker, Jolly good fellow \\
\hline Geek & Intellectual & Nerd, Cerebral, Egghead \\
\hline Original & Character & Pioneering, Novelty oriented, Original \\
\hline Soigné & Neat & Take care of himself, self-esteem \\
\hline
\end{tabular}

\section{Results}

\section{Standard photographs (Sorting Task)}

The various descriptive terms spontaneously expressed by the "naïve" panel were regrouped under generics and were further expressed as frequencies, allowing to be located in a $\mathrm{Y} / \mathrm{X}$ axis.

A statistical grouping according to social relationships (extroverted vs. introverted) and variable maturity allows to define 8 clusters as centroids (Figure 2). The "John Doe" group, seen as globally less mature, presents large overlaps with 4 other groups, making this adjective a rather commonly shared property, rather centered and globally more perceived as extroverted. The "NEET", "Bosses" and "Family Fathers" groups appear perceived as the more introverted psycho-social categories. In contrast, "Industrious" and "Dynamic Youngsters" are unsurprisingly perceived as more extroverted and judged of a lower maturity.

\section{Global perceptions of the II8 men by the "naïve" panel (Quiz Task)}

A PCA analysis (Figure 3), that explains $66.8 \%$ of data, allows the men to be positioned on a 2D map, based on the scores given for the 10 fixed verbatims previously mentioned. Of note, most men were centered on the PCA axis, i.e. that few were considered as extreme cases on the two axis. Of note, men judged older, tired and with a sensitive skin are more frequently found within the quadrant Classic / Intellectual.

However, on a hedonistic criterion, Figure 4 shows that, globally, the 60 women classify the 118 men through two major axis horizontal / Hedonism and vertical / Sympathetic appearance.

Accordingly, 4 groups can be identified, paired-opposed, illustrated by 4 different colors. 



Photos I (left) and 2 (right): Same subject photographed under the two modes (standard and natural).
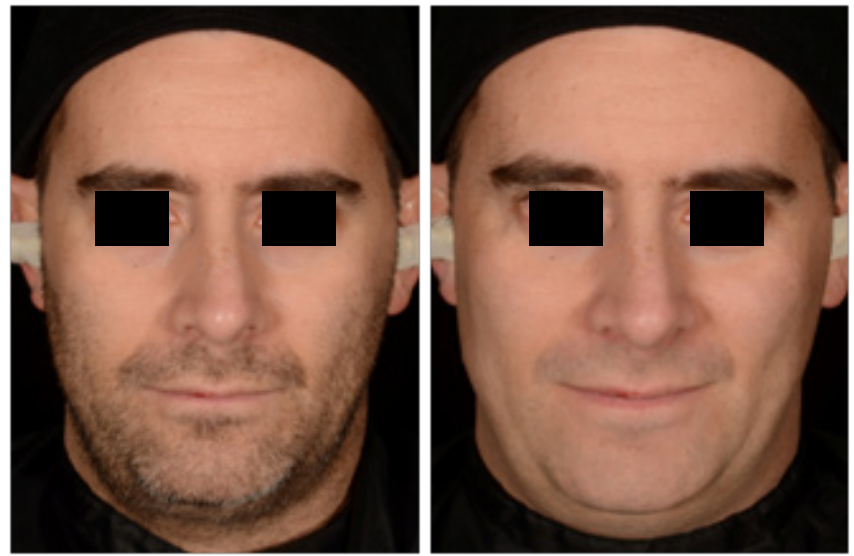

Photos 3 and 4: Standard photographs of the same subject with (left) and without (right) his beard ( 3 days w/o shaving).

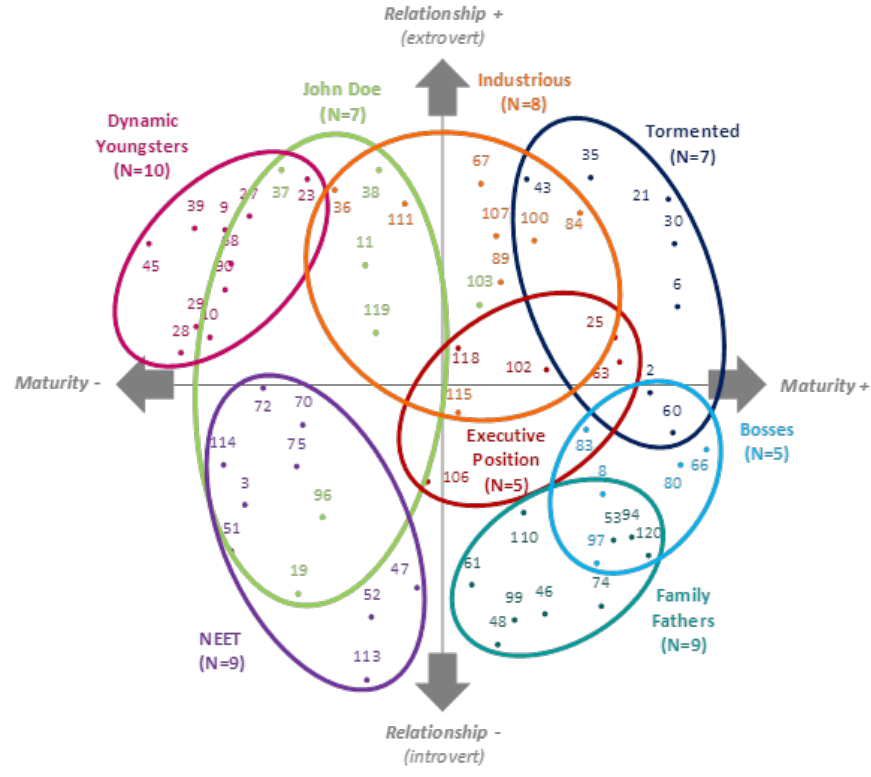

Figure 2 Positioning of the 8 perceived psycho-social clusters along the maturity and relationship attitude.

Group 1 (38\%): The less sympathetic men. They are mostly associated to Wise, Classic, Ambitious and Virile fixed verbatims. These men are generally viewed as Urban but Adventurous and judged older than the global cohort.

Group 2 (21.5\%): The more sympathetic men. These are viewed as Character, Gentle, Round and Neat. These men seem younger and are judged less tired than the global cohort.

Group3 (17.1\%): The more attractive men. These are mostly perceived as Character, Virile, Sportsman, Ambitious, Rounder, Neat and are found within the Adventurous category.

Group 4 (23.4\%): The less attractive men. These are perceived as Intellectual, with a sensitive skin. They are judged more tired and slightly older than the global cohort.

The PCA allows possible significant $(\mathrm{p}<0.05)$ inter-correlations (positive or negative) between the 10 fixed verbatims to be assessed. It indicates positive correlations between Sportsman/Virile/Ambitious/ Neat/Character. In contrast, Intellectuals are negatively correlated with Virile, Rounder, Ambitious and Character. This is shown by Table 3.

\section{Natural photographs (Sorting Task)}

A comparable grouping performed from these photographs shows a slightly different picture. This now leads to only 6 centroid clusters, as shown by Figure 5. The "John Doe" group (no evident characteristic) is unsurprisingly located at the center of the representation. "Family Fathers" appear logically more mature. "Social Sports men" are judged more extroverted and less mature, whereas the "Executive Position" are perceived as more introverted with a higher maturity. As seen before, the "John Doe" cluster appears rather well balanced along the two axis. The assessments of natural photographs, that integrate additional elements such as garments, hair style, thus afford a narrower definition of the clusters. This is illustrated by Figure 6 that gathers the displacements of the 8 initial clusters (standard photographs) to the 6 new clusters when shifting from standard to natural photographs.

Figure 6 shows different positioning of 2 clusters ("John Doe" and "Executive Position") whereas the "Dynamic Youngsters", "Family Fathers" and "Tormented" clusters do not show major changes. Of note, the "Bosses", "Industrious" and "NEET" clusters previously defined through standard photographs shows no particular positioning, i.e. disappearing from the picture in favor of a new "Social Sportsmen" group. In brief, the assessments of natural photographs by a "naïve" panel, as compared to standard ones, describe different psycho-social profiles. Interestingly, the "Tormented" subjects (that could be presumed introverted) are equally positioned on the psychosocial axis, although perceived as more mature.

\section{Influence of Beard \& Facial hair style upon assessments by the "naïve" panel (Quiz task)}

When shaved, the 40 men with beard appear differently assessed through standard photographs, as illustrated by Figure 7. The latter indicates that most subjects become displaced towards a more intellectual profile associated to an increased perception of a higher maturity. Some subjects show significant displacements (e.g. $\left.n^{\circ} 2,26,37\right)$ towards this trend, whereas a few others show an inverse displacement, albeit of small amplitude (e.g. $\mathrm{n}^{\circ} 11,14,23$, $27,31)$. Although the presence of beard seems to favor Sportsman, Virile, Character and Neat, its absence is now more mature, more 
Intellectual profiles, albeit at various extents according to subjects. Of note, shaving the beard mostly concerns the Sportsman/Virile vs. Intellectual axis and does not seem much influencing the perception on the axis Classic vs. Gentle.

Table 4 summarizes the influence of beard on the 10 fixed verbatims, expressed as difference between the means (with vs. without beard) through ANOVA analysis ( $<<0.05$ threshold). Table 4 shows that shaving leads to few significant changes (drops in red or increases in green). Shaving appears to favor a loss in "Virile" attribute whereas it gains in the "Neat" aspect.

However, it has to be acknowledged that, among psycho-socially perceived groups, shaving does not lead to many significantly different appreciations, although a gain in about one year in apparent age is noticed.

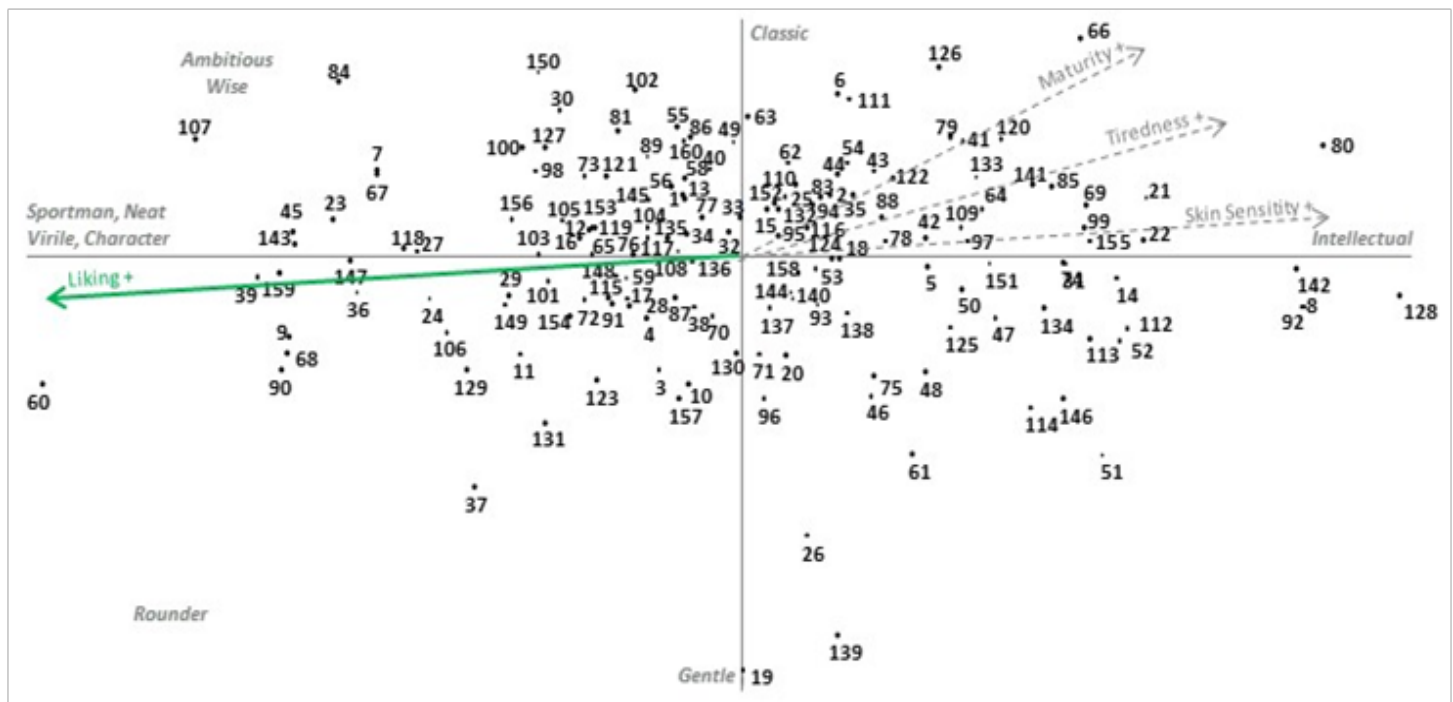

Figure 3 PCA of the repartition of the 118 men based on the scores given for the 10 fixed verbatims, defining two major axes (Classic-Gentle and Sportsman/ Virile-Intellectual) and their associated meanings (tired look, perceived age, sensitive skin).

Figure 4 PCA of the 118 men based on the scores given for the 10 fixed verbatims + HCA based on the coordinates, highlighting four groups according to



their attractive look and sympathetic look.

\section{Objective decoding of female "naïve" panel's perception when observing natural pictures of men}

Changes in the facial clinical signs in Caucasian males with age were previously described. ${ }^{17}$ The focus, here, was made on the facial signs that impact the perception of men faces by women. Table 5 gathers the six clusters defined on natural pictures (Figure 5), the significant impact of facial clinical signs in the clustering of male panel, i.e. clinical signs that are significantly higher or lower $(\mathrm{p}<0.01)$ in the considered cluster, as compared to all other parts of male population. In three clusters on six, only a significant impact of the studied facial signs has been noticed. For "Tormented" Glabellar wrinkles appear significantly more severe than all the other clusters $(+183 \%)$, whereas "Trendy Youngsters" and "Family fathers" present one sign significantly less severe than all the other clusters, 
for Glabellar wrinkles (-76\%) and Forehead wrinkles (-37\%), respectively. The importance of the signs of the upper half face, in this age cluster, in the overall face perception, is confirmed.

The same analysis was performed with instrumental measurements (Table 6) and leads to other observations with "Trendy Youngsters" presenting a lighter skin tone $(+8 \%)$ than all other clusters and "Executive Position" a less plumpness aspect $(+27 \%)$. "Social Sportsmen" present a skin more hydrated $(+24 \%)$ whereas "John Doe" show a darker skin tone (-4\%) and "Tormented" a less hydrated skin than other clusters (-35\%). "Family Fathers" are not separated from other clusters in any physical dimension investigated.

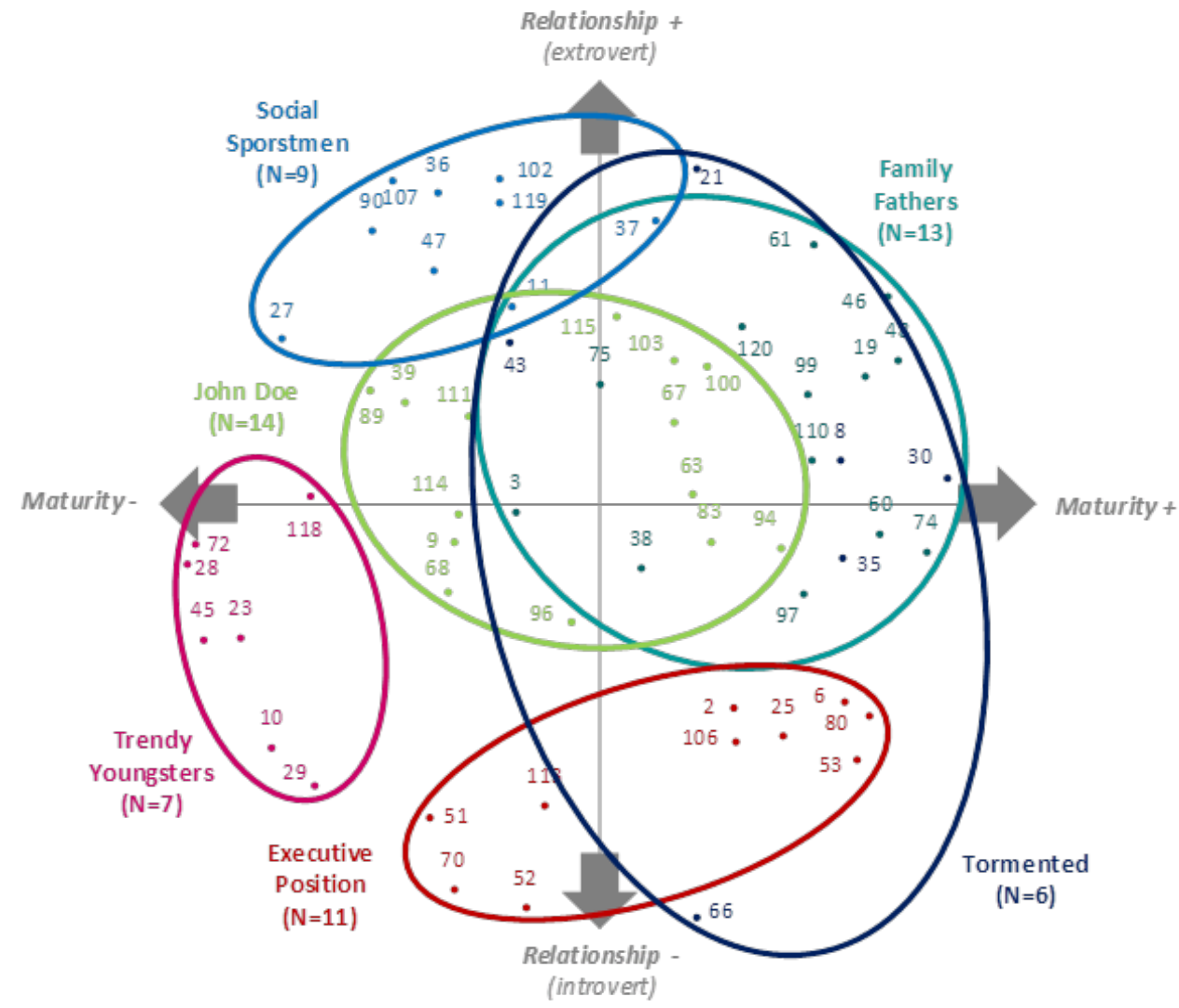

Figure 5 Positioning of the 6 clusters obtained from natural photographs.

Table 3 Correlations (negative/red or positive/green) between the fixed verbatims used by the "naïv" panel to describe the various profiles of the II 8 men.

\begin{tabular}{|c|c|c|c|c|c|c|c|c|c|c|}
\hline & Sportsman & Intellectual & Virile & Gentle & Rounder & Neat & Ambitious & Wise & Classic & Character \\
\hline \multicolumn{11}{|l|}{ Sportsman } \\
\hline Virile & 0.69 & $-0,63$ & & & & & & & & \\
\hline Gentle & 0.03 & -0.48 & -0.18 & & & & & & & \\
\hline Rounder & 0.11 & -0.76 & 0.20 & 0.75 & & & & & & \\
\hline Neat & 0.59 & -0.37 & 0.25 & 0.35 & 0.26 & & & & & \\
\hline Ambitious & 0.69 & -0.53 & 0.76 & -0.13 & 0.11 & 0.53 & & & & \\
\hline Wise & 0.20 & -0.31 & 0.35 & 0.04 & -0.03 & 0.31 & 0.60 & & & \\
\hline Character & 0.58 & $-0,78$ & 0.50 & 0.52 & 0,69 & 0.52 & 0.45 & 0.10 & -0.03 & \\
\hline
\end{tabular}




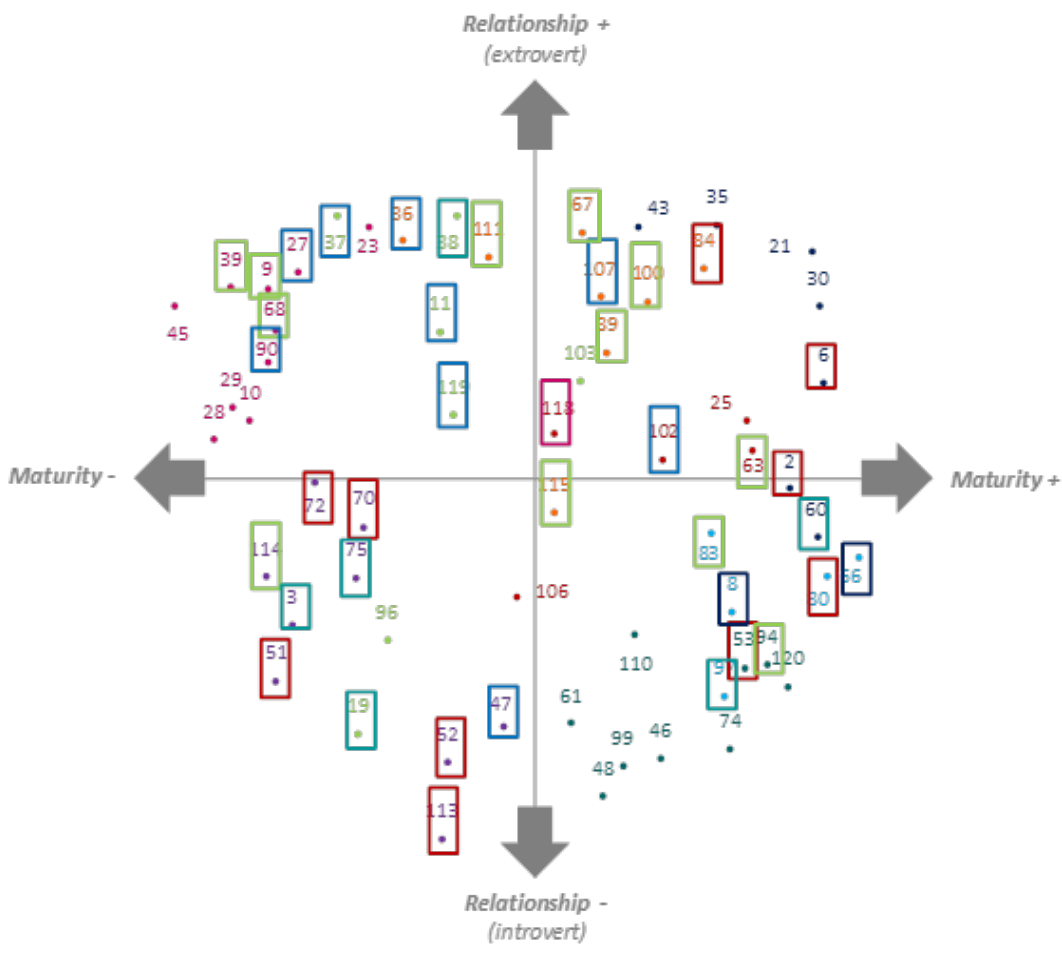

Figure 6 Different view of the positioning of the clusters when shifting from standard to natural photographs. In bold, natural photographs. Framed: men switching to another cluster when shifting from standard to natural photographs. The font color indicates the initial cluster (standard) and the frame indicates the new cluster (natural).

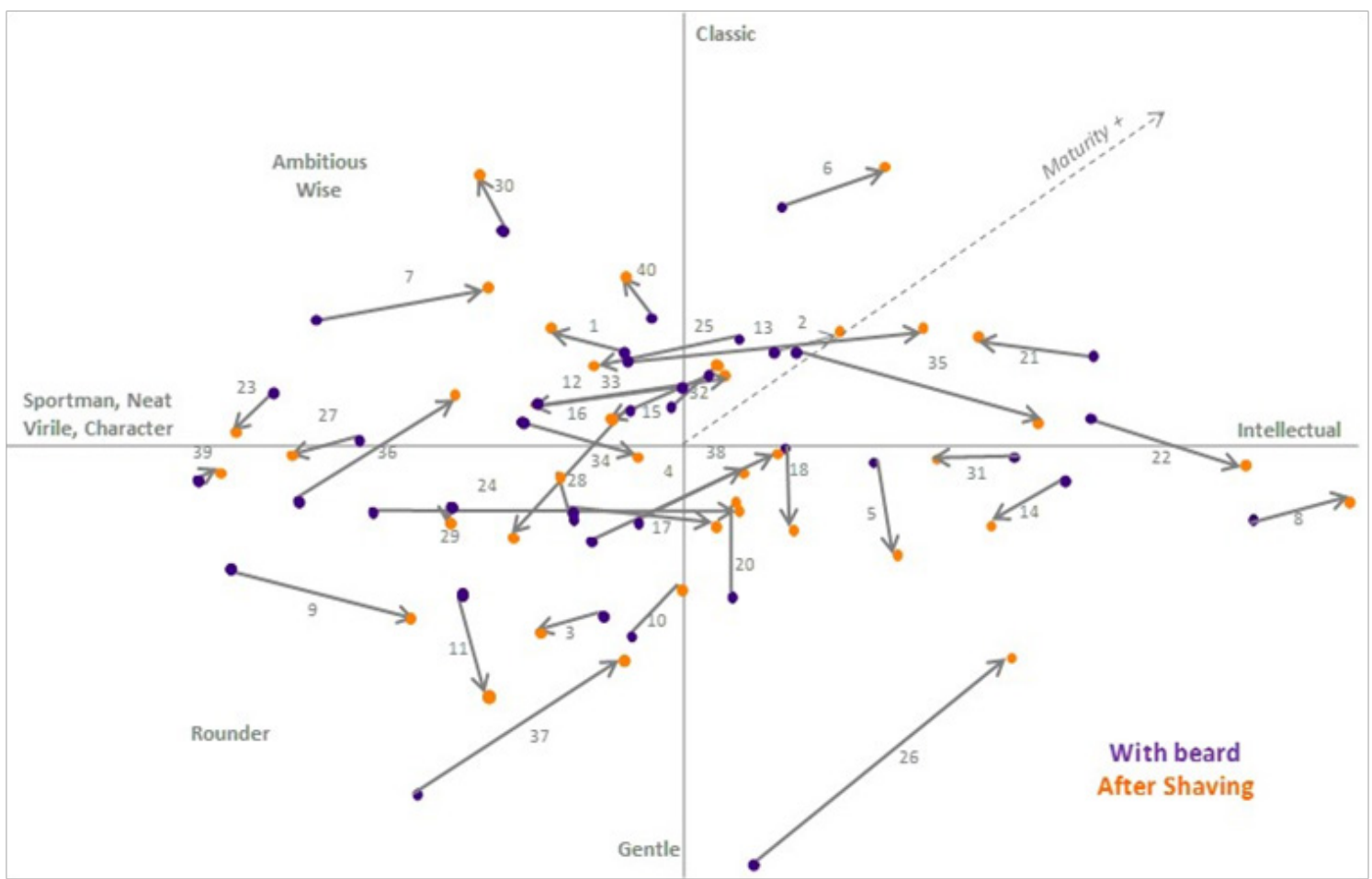

Figure 7 Displacements of assessed profiles of the 40 men with beard (in purple) and after shaving (in orange). 
Table 4 ANOVA analysis of changes perceived by the "naive" panel $(N=60)$ induced by shaving. Only significant changes $(\mathrm{p}<0.05)$ are presented either as positive (in green) or negative (in red).

\begin{tabular}{lll}
\hline \multicolumn{1}{c}{ Quiz criteria } & & Difference \\
Sportsman & & $\searrow-0.25$ \\
Intellectual & & $\nearrow 0.14$ \\
Virile & & $\searrow-0.43$ \\
Gentle & & $\nearrow 0.05$ \\
Rounder & & $\searrow-0.13$ \\
Neat & & $\nearrow 0,25$ \\
Ambitious & & $\nearrow 0.03$ \\
Wise & & $\nearrow 0.11$ \\
Classic & $\nearrow 0.01$ \\
Character & $\searrow 0.16$ \\
Liking & $\searrow-0.29$ \\
Perceived Age & $\nearrow 1.06$ \\
Tired look & $\nearrow 0.10$ \\
Sensitive skin & $\nearrow 0.19$ \\
\hline
\end{tabular}

\section{Discussion}

Taking into account the restricted size of the men cohort observed here, the results of the present study have to be taken with great caution and possibly represent the tip of the iceberg. They thus should be considered as qualitative observations obtained at a given time, in a given country, in a given culture (French men of almost all social categories). Addition of objective clinical and instrumental measurements of some facial signs (wrinkles/folds, sagging/ptosis, skin color, skin plumpness, skin hydration, sagging/ptosis.) may likely help to better explore the correspondence between perception and its spontaneously associated wordings.

At first, understanding the complex paradigm of wordings, their language translations, is by nature a highly complex task. Deciphering what an individual truly means by her/his chosen wording requires interpretations that may follow a fuzzy logic. This is a dilemma very often faced by MD's when attempting to cross objective data with the verbal assertions of their patients. "I have minor/medium/strong headaches", "I do not feel well, I feel so-so" are hardly measurable and comprehensive assessments.

Second, the present exploratory work aimed, as intellectual challenge, at determining the psycho-social perceptions of the faces of men by women through their verbal translations. The latter were here defined by various spontaneous wordings, that were arbitrarily summarized by "shortcuts" ("John Doe", "Executive Position", "NEET"). We acknowledge that these may be over-simplifying verbatims. Nevertheless, the protocol used here (standard vs. natural photographs) reveals the complexity of a spontaneous perception. Standard photographs, that only illustrate major facial traits (eyes, facial shape, nose, mouth, skin status) likely leave probably "room" to imagination, thereby creating 8 clusters. Natural photographs, that integrate additional elements (hair style, garments etc.) then restrict the psycho-social interpretations to 6 clusters, where "Bosses", "Industrious" and "NEET" became not expressed and favor the apparition of a new "Social Sportsmen" group. In brief, facial traits own dual aspects since their perception appears clearly influenced/ modulated by external fashion-related items (hair style, garments) and represent a major bias. These indeed show such permanent and rapid changes with short-live modes, that the results obtained here can only reflect an instant "snap".

Table 5 Impact of clinical facial signs in the perception of male face by female "naïv" panel on natural pictures. In bold green, sign significantly less severe in the cluster compared to all the other parts of population. In bold red, sign significantly more severe in the cluster compared to all the other parts of population.

\begin{tabular}{lllllll}
\hline & $\begin{array}{l}\text { Trendy } \\
\text { youngsters }\end{array}$ & $\begin{array}{l}\text { Executive } \\
\text { position }\end{array}$ & $\begin{array}{l}\text { Social } \\
\text { sportsmen }\end{array}$ & Family fathers & $\begin{array}{l}\text { John } \\
\text { doe }\end{array}$ & Tormented \\
\hline $\begin{array}{l}\text { Clinical } \\
\text { assessments }\end{array}$ & $\begin{array}{l}\text { Glabellar wrinkles } \\
\mathrm{P}=0.001 /(76 \%)\end{array}$ & 0 & 0 & $\begin{array}{l}\text { Forehead wrinkles } \\
\mathrm{p}=0.001 /(37 \%)\end{array}$ & 0 & $\begin{array}{l}\text { Glabellar wrinkles } \\
\mathrm{p}=0.001 /(+\mid 83 \%)\end{array}$ \\
\hline
\end{tabular}

Table 6 Impact of instrumental facial measurements in the perception of male face by female "naïv" panel on natural pictures. In bold green, physical parameter significantly higher in the cluster compared to all the other parts of population. In bold red, physical parameter significantly lower in the cluster compared to all the other parts of population.

\begin{tabular}{lllllll}
\hline & $\begin{array}{l}\text { Trendy } \\
\text { Youngsters }\end{array}$ & $\begin{array}{l}\text { Executive } \\
\text { Position }\end{array}$ & $\begin{array}{l}\text { Social } \\
\text { Sportsmen }\end{array}$ & $\begin{array}{l}\text { Family } \\
\text { Fathers }\end{array}$ & John Doe & Tormented \\
\hline $\begin{array}{l}\text { Instrumental } \\
\text { measurements }\end{array}$ & $\begin{array}{l}\text { Lightness } \\
\mathrm{p}=0.00 \mathrm{I} /(+8 \%)\end{array}$ & $\begin{array}{l}\text { Plumpness } \\
\mathrm{p}=0.00 \mathrm{I} /(+27 \%)\end{array}$ & $\begin{array}{l}\text { Hydration } \\
\mathrm{p}=0.0 \mathrm{I} /(+24 \%)\end{array}$ & 0 & $\begin{array}{l}\text { Lightness } \\
\mathrm{p}=0.003 /(4 \%)\end{array}$ & $\begin{array}{l}\text { Hydration } \\
\mathrm{p}=0.0 \mathrm{I} /(35 \%)\end{array}$ \\
\hline
\end{tabular}

From a hedonistic viewpoint, attractiveness and sympathetic aspect seem ruling the appreciations by the "naïve" panel. The perception of sympathy seems driven by Character, Gentle, Rounder, Neat attributes and gather profiles perceived as younger and less tired. Attractiveness seems giving privilege to the perceptions of Character, Virile, Sportsmen, Ambitious, Rounder and Neat and gather men ranked among the "Adventurous" category.
The Analysis of objective data collected in the six clusters formed by the views of natural pictures, allowed to find some significant link between female perception and men facial signs. These comprise wrinkles (Glabellar wrinkles \& Forehead wrinkles), skin tone lightness, skin plumpness or skin hydration - all connected to the facial appearance. Some interpretations seem obvious such as "Tormented" bearing more severe Glabellar wrinkles, "Trendy Youngsters" with 
lighter skin tone and less severe Glabellar wrinkles and "Executive Position" with a more flaccid skin and less plump whereas the three other clusters present objective point of differentiation that needs to be translated.

Beard (or moustache/facial hair style), a so typical male attribute, clearly influences the psycho-social perception of men by women. Beard can hardly be summarized as body hairs on cheeks or chin. It owns deep, cultural, historical or religious roots. It is often viewed, at least in the western culture, as a symbol of wisdom/maturity/ superiority, iconic representations of God included. Some religions or cultures even make beard an imperative coding attribute (Muslims, Catholic Orthodox's, Sikhs, Amish).

Wearing a beard, in the western culture, follows fast moving fashions. Here, despite the rather low number of observations (40 subjects), the (provisory) absence of beard seems conferring to the same men a more mature or an intellectual psycho-social profile, irrespective with other signs of fashion (standard photographs). Interestingly, although beard is logically perceived as a virile trait, its absence makes his owner to become more neat and younger by about one year. Despite some positive assets, beard was negatively perceived as a less neat - or clean - facial feature of the studied men. This suggests a deeply anchored cultural understanding, at least among Caucasian women, that was not explored in the present work, as out of its scope.

The present work has obvious limitations as it only dealt with Caucasian women and men. It however depicts how men facial appearance is more strongly driven by cultural, social, hedonistic characteristics rather than strict aesthetical criteria ("pretty eyes, mouth or nose, nice hairs etc.). It remains clear that this work calls for its extension to other cultures or ethnics. Worldwide, the mutual relationships between men and women are anchored within so many cultural or religious codes, coupled to the subtleness of their specific verbal expressions. Different facial traits, rarity or abundance of beard in some ethnic or religious groups, different skin color, different garments, different cultural standards of attractiveness or intimate perceptions are paramount factors. All these criteria compose a so large human mosaic that the approach presented here has to be widen to other socio-cultural-ethnic human groups.

\section{Acknowledgments}

Authors want to thank Mr Didier Saint-Leger, Mr Roland Bazin, Mr Olivier Coubard, Mrs Sophie Canevet-Zaida, Mr Thierry Cotton, Mrs Caroline Negre, Mrs Johanna Caron, Mr Matthieu Cassier and $\mathrm{Mr}$ Thierry Lageat not only for their great help in completion of this paper and global study but also for their strong support and enthusiasm.

\section{Conflicts of interest}

The authors report no conflicts of interest.

\section{Funding}

The present work was entirely funded by the L'Oréal Research \& Innovation Department.

\section{References}

1. Little AC, Roberts CS. Evolution, appearance and occupational success. Evol Psychol. 2012;10(5):782-801.
2. Von Stockhausen L, Koeser,Sczesny S. The gender typicality of faces and its impact on visual processing and on hiring decisions. Exp Psychol. 2013;60(6):444-452.

3. Isaac $\mathrm{C}$, Lee $\mathrm{B}$, Carnes $\mathrm{M}$. Interventions that affect gender bias in hiring: a systematic review. Acad Med. 2009;84(10);1440-1446.

4. Greven IM, Downing PE and Ramsey R. Linking person perception and person knowledge in the human brain. Soc Cogn Affect Neurosci. 2016;11(4):641-651.

5. Vashi NA, de Castro Maymone MB, Kundu RV. Aging differences in ethnic skin. J Clin Aesthet Dermatol. 2016;9(1):31-38.

6. Bulpitt C, Markowe HL, Shipley MJ. Why do some people look older than they should? Postgrad Med J. 2001;77(911):578-581.

7. Mayes AE, Murray PG, Gunn DA, et al. Environmental and lifestyle factors associated with perceived facial age in Chinese women. PLoS One. 2010;5(12):e15270.

8. Dibeklioglu H, Alnajar F, Ali Salah A, et al. Combining facial dynamics with appearance for age estimation. IEEE Trans Image Process. 2015;24(6);1928-1943.

9. Levakov A, Vuckovic N, Doali M et al. Age-related skin changes. Med Pregl. 2012;65(5-6):191-195.

10. Hourblin V, Nouveau S, Roy $\mathrm{N}$ et al. Skin complexion and pigmentary disorders in facial skin of 1204 women in 4 Indian cities. Indian $J$ Dermatol Venereol Leprol. 2014;80(5):395-401.

11. Qiu H, Flament F, Long X, et al. Seasonal skin darkening in Chinese women: The shanghaiese experience of daily sun protection. Clin Cosmet Investig Dermatol. 2013;6:151-158.

12. Shaiek A, Flament F, François G, et al. A new tool to quantify the geometrical characteristics of facial skin pores. Changes with age and a make-up procedure in Caucasian women. Skin ResTechnol. 2017;23(2):249-257.

13. Bazin R, Doublet E. Skin Aging Atlas. Volume 1, Caucasian Type. Paris: Editions Med Com. 2007.

14. Bazin R, Flament F. Skin Aging Atlas. Volume 2, Asian Type. Paris: Editions Med Com. 2010

15. Bazin R, Flament F, Giron F. Skin Aging Atlas. Volume 3, Afro-American Type. Paris: Editions Med Com. 2012

16. Bazin R, Flament F, Rubert V. Skin Aging Atlas. Volume 4, Indian Type. Paris: Editions Med Com. 2015.

17. Flament F, Amar D, Feltin C, et al. Evaluating age-related changes of some facial signs among men of four different ethnic groups. Int $J$ Cosmet Sci. 2018;40(5):502-515.

18. Fink B, Matts PJ, Emiliano D, et al. Colour homogeneity and visual perception of age, health and attractiveness of male facial skin. J EurAcad Dermatol Venereol. 2012;26(12):1486-1492.

19. Fink B, Bunse L, Matts PJ, et al. Visible skin colouration predicts perception of male facial age, health and attractiveness. Int J Cosmet Sci. 2012;34(4):307-310.

20. Fink B, Matts PJ, Brauckmann C, et al. The effect of skin surface topography and skin colouration cues on perception of male facial age, health and attractiveness. Int J Cosmet Sci. 2018;40(2):193-198.

21. Bernard BA, Saint-Léger D, Leclaire J. The multifaceted human facial appearance: beyond skin and hair. J Dermat Cosmetol. 2018;2(2):147-154.

22. Bazin R, Fanchon C. Equivalence of face and volar forearm for the testing of moisturizing and firming effect of cosmetics in hydration and biomechanical studies. Int J Cosmet Sci. 2007;28(6):453-460. 
23. Flament F, Bazin R. Influences of age, ethnic group, and skin sites on a provisory skin marking, experimentally induced, in vivo. Skin Res Technol. 2018;24(2):180-186.

24. Flament F, Gautier B, Benize AM, et al. Seasonally-induced alterations of some facial signs in Caucasian women and their changes induced by a daily application of a photoprotective product. Int J Cosmet Sci. 2017;39(6):664-675.
25. Qiu H, Long X, Ye C, Flament F et al. Instrumental and clinical studies of the facial skin tone and pigmentation of Shanghaiese women. Changes induced by age and a whitening product. Int J Cosmet Sci. 2012;34(1):4954. 